\title{
Usability of Overview-Supported Zooming on Small Screens with Regard to Individual Differences in Spatial Ability
}

\author{
Thorsten Büring \\ University of Konstanz \\ buering@inf.uni- \\ konstanz.de
}

\author{
Jens Gerken \\ University of Konstanz \\ gerken@inf.uni- \\ konstanz.de
}

\author{
Harald Reiterer \\ University of Konstanz \\ reiterer@inf.uni- \\ konstanz.de
}

\begin{abstract}
While zoomable user interfaces can improve the usability of applications by easing data access, a drawback is that some users tend to become lost after they have zoomed in. Previous studies indicate that this effect could be related to individual differences in spatial ability. To overcome such orientation problems, many desktop applications feature an additional overview window showing a miniature of the entire information space. Small devices, however, have a very limited screen real estate and incorporating an overview window often means pruning the size of the detail view considerably. Given this context, we report the results of a user study in which 24 participants solved search tasks by using two zoomable scatterplot applications on a PDA - one of the applications featured an overview, the other relied solely on the detail view. In contrast to similar studies for desktop applications, there was no significant difference in user preference between the interfaces. On the other hand, participants solved search tasks faster without the overview. This indicates that, on small screens, a larger detail view can outweigh the benefits gained from an overview window. Individual differences in spatial ability did not have a significant effect on task-completion times although results suggest that participants with higher spatial ability were slowed down by the overview more than low spatial-ability users.
\end{abstract}

\section{Categories and Subject Descriptors}

H.5.2 [Information Interfaces and Presentation]: User Interfaces - Graphical User Interfaces

\section{General Terms}

Human Factors, Design

\section{Keywords}

small screen, zoom, spatial ability, scatterplot, overview plus detail

Permission to make digital or hard copies of all or part of this work for personal or classroom use is granted without fee provided that copies are not made or distributed for profit or commercial advantage and that copies bear this notice and the full citation on the first page. To copy otherwise, to republish, to post on servers or to redistribute to lists, requires prior specific permission and/or a fee.

\section{INTRODUCTION}

This paper analyzes the usability of zoomable (ZUIs) and overview plus detail $(\mathrm{o}+\mathrm{d})$ interfaces on small screens, taking into account individual differences in spatial ability. There are two main objectives of this research. First, we wanted to explore how an overview affects user performance, satisfaction and interface preference, given a very limited screen real estate. The second objective was to investigate how users with different levels of spatial ability interact with interfaces that offer different amounts of overview information. The results obtained may support developers when designing interfaces for small devices.

\section{RELATED WORK}

The following section summarizes relevant previous research on ZUIs, o+d interfaces and spatial ability. It also explains our motivation and design considerations for the experiment conducted.

\subsection{Zoomable User Interfaces}

Today's information age is characterized by large amounts of data that need to be processed not only by machines but also by humans. While there is rapid advancement in hardware capacity and performance, the human bandwidth of perception remains rather static. The management of scale has thus become a major challenge for user interface designers. ZUIs can ease access to large-scale data and, due to recent zoom applications like Google Earth [17], are becoming increasingly popular beyond the scientific community. ZUIs are based on the assumption that navigation in information spaces is best supported by tapping into our natural spatial and geographic ways of thinking [21]. In order to implement this approach on a computer screen, data objects must be organized in space and scale. Users can interact directly with the information space by performing panning and zooming operations. Since a ZUI lets the user view much more information than can normally fit on a single screen, it thus provides a valuable solution for enhancing limited screen real estate.

Different zoom techniques need to be distinguished. Many map-based interfaces feature a purely geometric zoom. Zooming in on an object of interest (e.g. a street corner) the relevant map clipping simply becomes magnified. Another approach is semantic zooming. In this case, information ob- 
jects are given a different visual representation depending on the amount of real estate available to them [21]. Semantic zooming has been incorporated into various experimental PDA systems. In a calendar application called DateLens, dates are organized in a tabular display where each row represents one week, with seven columns representing the days of the week. Tapping on a cell causes the cell to expand and to reveal more detailed information about the corresponding date. Based on a fisheye algorithm the other cells shrink accordingly [3]. A similar approach was used for providing multiple views of application data [18] and for presenting web pages on small screens [19]. The latter system displays web pages as a thumbnail view in which the page layout is enhanced by fragments of readable text. On zooming in, the abbreviated text is replaced by the complete text. One PDA application that includes both semantic and geometric zoom techniques is ZuiScat [4]. It features a zoomable scatterplot tool to visualize abstract information spaces and will be discussed in more detail in section 3.4.

While research has shown that ZUIs are in many cases superior to conventional interfaces [3, 19], the main drawback is that some users tend to lose their overview while navigating $[5,15]$. These orientation problems can be reduced by smooth transitions between scale levels [5, 23], but a more powerful approach to preserving the user's sense of position and context is the use of $\mathrm{o}+\mathrm{d}$ interfaces.

\subsection{Overview plus Detail Interfaces}

$\mathrm{O}+\mathrm{d}$ interfaces are characterized by a multi-window layout, where one window is used to present details while the other one gives an overview of the information space [22]. We can distinguish between overviews offering interaction possibilities like panning and scaling, and non interactive overviews which just help users to orientate. Determining the dimensions of the two windows can strongly affect the usability of applications for small devices. The larger the overview the more information can be displayed and the easier it is for the user to navigate in it. On the other hand, a larger overview means a smaller detail window, hence it becomes more difficult for the user to access information objects due to congestion. In other words, there exists a trade-off between the amount of overview information provided and the size of the detail view. This problem applies to the most common side-by-side layout of the two windows. Alternative display techniques like transparent or on-demand overviews require less screen space, but typically add a significant amount of potentially-disturbing complexity to the interface [23, 22].

$\mathrm{O}+\mathrm{d}$ interfaces on small screens have not been researched as yet, but a number of empirical studies have investigated the usability of overviews on desktop computers. Results indicate that users in most cases prefer an $\mathrm{o}+\mathrm{d}$ interface over other interfaces, while the performance varies with the type of information space used and the type of tasks examined. For instance, a study that compared the usability of a linear, a fisheye, and an o+d interface for electronic documents [16] found that essays written using the $o+d$ interface received higher grades while subjects were faster at reading and answering questions using the fisheye view. All but one subject preferred the $\mathrm{o}+\mathrm{d}$ interface. Another study analyzed the same visualization techniques for a web browser [2]. The authors discovered that the average performance of the fish- eye view for a set of four different task types proved to be superior to the other two interfaces. Still, subjects preferred the $\mathrm{o}+\mathrm{d}$ interface. One study also compared the usability of zoomable user interfaces with and without overviews [15]. Subjects solved browsing and navigation tasks on two maps, where one map was organized in multiple levels and thus allowed for semantic zooming. Again, users preferred the $\mathrm{o}+\mathrm{d}$ interface, though subjects were faster without the overview when using the map with multiple levels. The authors assumed that this type of information space provides richer navigation cues due to its semantic organization and thus renders an overview rather unnecessary. The study also found a very high variance in the subjects' ability to navigate the map, in task completion times and in accuracy. This observation indicates that individual differences may have a strong influence on user performance in ZUIs and $\mathrm{o}+\mathrm{d}$ interfaces.

\subsection{Spatial Ability}

Spatial ability, which can be defined as the ability to generate, retain, retrieve, and transform well-structured visual images [20], is often cited as being one of the best predictors of human-computer performance $[11,12,10]$. One study, for example, examined individual differences among users of a hierarchical file system and found that high-spatial users completed tasks more quickly than low-spatial users who tended to become lost in the file structure [26]. In a later study, it was shown that the differences between individuals could be eliminated when a hierarchical one-layer-view navigation was replaced by a visual mediator such as, in this case, an overview of the complete navigation hierarchy with no hidden layers [24]. The authors argued that the more visual interface compensated for the inability of low-spatial users to construct a mental model of the information space. The same cognitive mechanism may apply to ZUIs. While users zoom in, information objects move out of sight and thus fewer orientation cues are displayed. Without an overview, users are forced to either rely on their mental model of the information space or to perform repetitive and time consuming zoom-out operations to reorient themselves. Obviously, when it comes to task-completion time, such a system benefits high-spatial users. This hypothesis would also correspond with the results of another study that analyzed usability issues of $2.5 \mathrm{D}$ visual user interfaces [9]. It found that high spatial-ability users were significantly faster in initially navigating and finding search items in virtual reality systems that require a 'fly-through' with the mouse. The lower their spatial ability, the more users were likely to become lost and the fewer search tasks were completed [8].

While visual interfaces can improve the performance of lowspatial individuals, they may, on the other hand, hinder high-spatial users. A study that focused on spatial scanning and perceptual speed investigated the influence of spatial abilities on the interaction with digital library interfaces [1]. As it turned out, individuals with high spatial abilities performed worst when using a word map - i.e. a two-dimensional map of the 100 most frequent word roots reflecting the intrinsic structure of the bibliographic collection. Similar results were found for semantic search spaces: high-spatial users performed better in a plain textual interface for a document collection than in a spatial user interface $[7,6]$. While this effect has not been fully clarified, it may 
however give a hint as to why overview windows can, in some cases, degrade user performance.

Overall, the available literature indicates that it is difficult to predict the effect specific design features would have on users with different cognitive abilities (e.g. [27, 8]). Hence, the need for further research into these issues is strongly indicated.

\section{EXPERIMENT}

The contribution of this paper is to investigate the usability of overview-supported ZUIs on small screens. For this purpose, we conducted an experiment in which participants had to complete search tasks on a PDA using two zoomable scatterplot applications - one that featured an overview (overview interface) and one that relied solely on the detail view (detailonly interface). To analyze the effect that individual differences in spatial ability would have on user performance and interface preference, participants had to complete a psychometric test. This section describes our hypotheses and experimental parameters.

\subsection{Hypotheses}

The hypotheses were:

1. Users would prefer the overview over the detail-only interface because of the additional orientation and navigation features provided. This hypothesis was based on the research results discussed in section 2.2. We expected that the overview would give the users a feeling of control [23] and thus compensate for the smaller size of the detail view, which was still manageable for the users, thanks to the ZUI.

2. Task-completion time would be better for the detailonly interface because of the rich orientation cues given by the scatterplot labels. It was assumed that overview facilities can become a hindrance when the information space searched by users already provides extensive orientation cues [15]. Moreover, visual switching between the two windows may slow users down [2].

3. Users with low spatial ability would have a longer taskcompletion time across interfaces than participants with higher spatial ability. This hypothesis was based on the research findings discussed in section 2.3.

4. The overview interface would reduce the performance difference between high and low-spatial participants. We expected that users who were not able to build up a mental model of the information space, would have to reorient themselves more frequently. Having an overview may eliminate the need for a mental model and hence support low-spatial users in catching up on task-completion times.

\subsection{Participants}

For the study we selected 24 subjects, 12 males and 12 females. All of them were students at the University of Konstanz. Their fields of study varied greatly, however none of the subjects studied computer science. The mean age was 24 years, ranging from 19 to 30 years. As revealed by the pretest questionnaire, none of the participants owned a PDA, although eight had already used one and were therefore familiar with the general interaction concept. All of them were familiar with a PC and used it daily. We also asked our participants if they were familiar with Google Earth. Twenty-one had at least heard of it and fourteen used it from time to time, but not regularly.

\subsection{Materials}

The study was run on a Hewlett-Packard iPAQ hx4700 Pocket PC with Windows Mobile 2003. The device featured a $624 \mathrm{MHz}$ processor, $64 \mathrm{MB}$ SDRAM and an interpolated 480x640/64K color VGA touchscreen. A digital video camera recorded the screen of the iPAQ; interactions like zooming and panning were automatically logged on the device. To measure user satisfaction we used the Attrakdiff questionnaire [13]. It uses a 7-point semantic differential and has 28 items in total and 7 for each dimension. Since the dimensions appeal and hedonic quality (identity and stimulation) were of less interest, we only used the dimension pragmatic quality (PQ), which tries to measure the user satisfaction regarding functionality. As a psychometric test we used 5 subtests of the Leistungspruefsystem (LPS) developed by Horn [14]. LPS is an intelligence test which measures the seven primary mental abilities as defined by Thurstone [25]. We used subtests 7-10 to measure the spatial ability and subtest 14 to measure perceptual speed. The latter was only used to test for unwanted correlations between spatial-ability scores and the perceptual speed. System preference and overview usage were obtained with post-test questionnaires; demographical information as well as PDA knowledge, computer experience, etc. with a pre-test questionnaire.

\subsection{Interfaces}

We believe that zoomable scatterplot interfaces have a great potential on small devices and thus we were mainly interested in analyzing orientation issues for abstract information spaces. However, the tasks analyzed are not specific to abstract data and hence the results of the experiment may be generalizable to more physical domains such as, for instance, maps. For the experiment, we implemented two Pocket PC applications using the Microsoft .NET compact framework 1.1. Both interfaces are based on the ZuiScat visualization [4] and present a movie database with 85 items.

\subsubsection{Detail-only}

The detail-only interface showed a scatterplot diagram in which each movie in the database was represented by a small rectangle and positioned according to the scatterplot axes of popularity rating $(\mathrm{X})$ and year of release $(\mathrm{Y})$ (Figure 1 a). To retrieve exact attribute values for a single movie, users tapped in the vicinity of the corresponding rectangle in the diagram. The system responded by highlighting the item which was closest to the pen position. Highlighting, in this case, means that the border of the rectangle was drawn red and enhanced with additional grid lines and data labels (Figure 1b). If the rectangle was overlapped by another item, it was moved to the foreground. To reduce congestion and to access movie information, users tapped and held the pen down. A fluent zoom animation was triggered after an initial delay of 150 milliseconds. While zooming in, the highlighted item moved smoothly to the center of the screen and the magnified rectangles became display areas for the posters of the movies they represented (Figures 1b, 
c). Users could interrupt the zoom operation any time by lifting the pen. If not interrupted, the animation continued until the highlighted rectangle filled the entire window space. At that point, the poster zoomed back out (anti-zoom) and made room for textual movie information to appear. The rectangle had changed its representation into a record card (Figure 1d). To return to the diagram view and to zoom out, users pressed the button at the bottom of the screen. The record card grew smaller and changed its representation accordingly. Again, users could stop the operation by lifting the pen, otherwise the animation continued until the default scale of the diagram had been reached. Zooming in from the default scale to the maximum zoom level took 1.8 seconds and magnified the information space 40 times. Users could also pan the diagram by dragging it with the stylus.

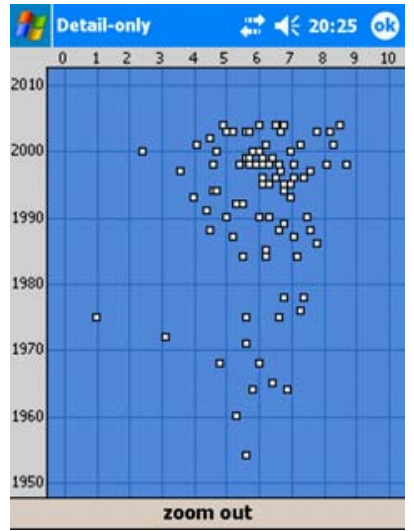

(a)

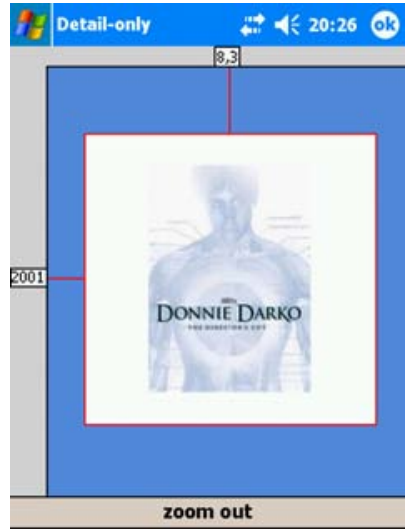

(c)

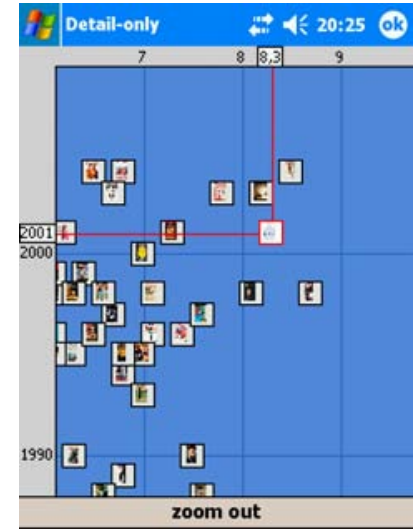

(b)

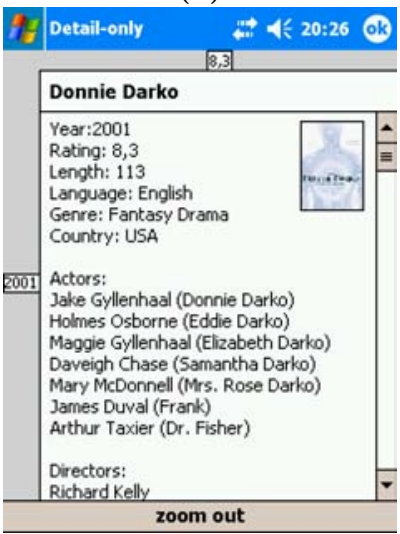

(d)
Figure 1: Detail-only interface: (a) default scale, (b, c) fluent zoom, (d) content view.

\subsubsection{Overview}

The second interface enhanced the detail view with a smaller overview window at the bottom of the screen (Figure 2a). The size of the overview was chosen such that the same scatterplot scale intervals as in the detail view could be used, with the axes labels remaining legible. This resulted in a layout in which the detail view was 40 percent smaller than in the detail-only interface. Both windows were tightly coupled, i.e. performing an operation on one view immediately updated the other one. The overview featured a field-ofview box (yellow rectangle) denoting the clipping currently presented in the detail view. Items that were highlighted on the detail view appeared red on the overview. Users could either pan on the overview by dragging the field-of-view box, or jump to another position by tapping the overview window outside the box. The field-of-view box then automatically moved to the pen position. If users had previously retrieved a movie's textual representation on the detail view (Figure $2 \mathrm{~b}$ ), panning or jumping on the overview changed the zoom level from 40 to 32 such that the detail window switched back to the diagram view. Zooming on the overview was achieved by first tapping on the scale button and then drawing a new field-of-view box on the overview. The box had a fixed aspect ratio which was determined by the ratio of the views. By zooming on the overview, it was not possible to achieve a scale level of over 32 . Hence, to retrieve an item's textual representation, users had to zoom on the detail view.

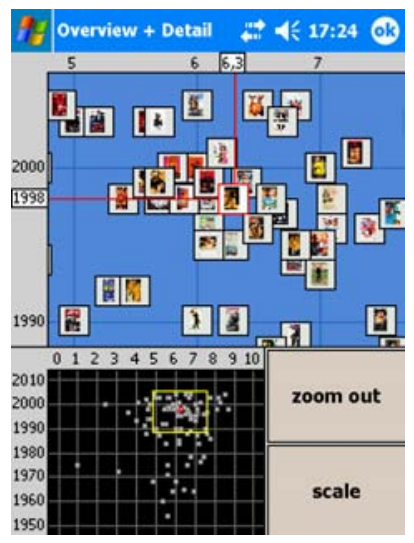

(a)

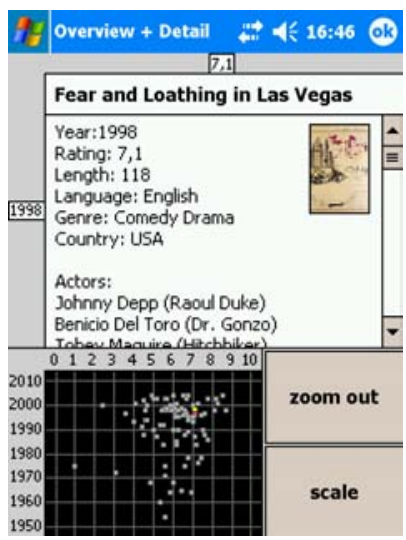

(b)
Figure 2: Overview interface: orientation and navigation features.

\subsection{Tasks}

During the experiment, for each of the two interfaces users had to solve a set of 12 tasks. A task set comprised four questions for each of the three following task types:

- Visual Scan

For example: how many movies in the collection have been produced after the year 2000 and have a popularity rating greater than or equal to 6 ?

- Information Access

Who is the director of the movie with a popularity rating of 4.4 ?

- Comparison of information objects

Which of the two movies from 1990 with the popularity ratings 6.3 and 6 is longer?

The task questions were written in German, the participants' native language.

\subsection{Experimental Design}

We used a counter-balanced within-subjects design, balancing the two interface types and task sets. This resulted in four different groups mirroring all possible variations of interface and task set order. We randomly assigned six subjects to each group. For analysis, we mainly used repeated 
measures ANOVAs (RM-ANOVAs) and regression analysis. Our independent variables were interface type (overview and detail-only) and spatial ability (used as both LPS C-score and dichotomized group variable). The dependent variables were task-completion time (in seconds), system-preference (overview and detail-only), user-satisfaction (Attrakdiff PQ Scores), error-rate (number of incorrect answered tasks), and navigation-actions (panning and zooming attempts and distances).

\subsection{Procedure}

Trials started with the participants answering a pre-test questionnaire focusing on demographic data and familiarity with computers, PDAs and ZUIs. Next, subjects had to complete the spatial-ability test, which took about 20 minutes (14 minutes test time) for all 5 modules. After that a short break was offered. When the participants indicated that they were ready to proceed, the test administrator gave a short introduction on how to use a PDA and handed the device over to the participants. Once the first of the two applications had been loaded, a tutorial movie about the interface was shown. At certain points, the test administrator paused the movie and asked subjects to reproduce operations on the PDA. Support was given as needed. When participants showed that they had understood the interface, each of the twelve task questions was presented to them successively as a printout. Participants read the question aloud and then pressed a button on the PDA screen to display the scatterplot interface, which they then navigated as necessary. When they felt they could answer the question, users tapped the cross button at the top-right corner of the interface. The scatterplot was then hidden and users answered aloud. During tasks sessions the test administrator was not allowed to provide any support. Having completed the first task set, subjects answered the Attrakdiff questionnaire. Subsequently, the same procedure was repeated for the second interface and the second task set. The experiment ended with participants answering a preference questionnaire and receiving a movie theater voucher worth $€ 10$. Experiment sessions lasted on average about one hour.

\section{RESULTS}

The following section describes the results of our study. We will first focus on the four hypotheses and then present some additional analysis.

\subsection{H1: Interface Preference}

In our first hypothesis we assumed that users would prefer the overview interface over the detail-only interface. However, analysis revealed that only 10 subjects preferred the overview interface compared to 13 favoring the detail-only interface. One subject was unsure. While the difference is not significant, $X^{2}(1, N=23)=0.391, p<0.532$, it nevertheless contradicts our hypothesis. Analyzing the users statements, we were able to identify two main reasons for this result:

- Most of the subjects who were in favor of the detailonly interface stated that they preferred the larger size of the detail window. They found the system easier to use and preferred not having to decide which way to solve a task. (8 subjects)
- Furthermore, some users stated that they had problems with zooming and panning in the overview. Due to the small size of the window, they found these functions rather imprecise and difficult to use. (5 subjects) Our observations underlined these statements, especially regarding the scale functionality. Most subjects tried to draw bounding boxes in a different aspect ratio than the predefined one. As mentioned in 3.4.2, this was not possible and some users became irritated by being unsure of when or how to use these additional navigation features.

Interestingly, five of the users who preferred the detail-only interface stated that they liked the overview window as an orientation help but either did not find the interaction possibilities useful or encountered problems using them. They therefore voted for the detail-only interface. This could indicate that a longer training phase might increase the acceptance of an overview window, because users would then become more certain which overview function is useful at a given time, and how to use it.

\subsection{H2: Task-Completion Time}

In our second hypothesis we assumed that subjects would be able to solve tasks significantly faster using the detail-only interface. It took our subjects on average 379.34 seconds to complete all 12 tasks with the detail only interface but 452.65 seconds with the overview interface (Figure 3 ). This difference is highly significant, $F(1,23)=16.5, p<0.001$, and therefore supports our hypothesis. A detailed analysis of the three different task types reveals that, for all of them, subjects were significantly faster using the detail-only interface (task type 1: $F(1,23)=7.587, p<0.05$; task type 2 : $F(1,23)=7.569, p<0.05$; task type $3 F(1,23)=5.797, p<$ $0.05)$.

We also analyzed which of the interaction possibilities had the greatest influence on task-completion times. A stepwise regression analysis revealed that, for the detail-only interface, zooming attempts and panning attempts were the best predictors for task-completion time. Together they explained about 56 percent of the variance (ANOVA results: $F(2,21)=13.488, p<0.001)$. Panning distance was also nearly as good a predictor as panning attempts (also 56 percent explained variance). Regarding the overview interface, here again zooming attempts within the detail window and, in this case, panning distance in the detail window were the best predictors, explaining about 64 percent of the variance (ANOVA results: $F(2,21)=18.525, p<0.001$ ). So regarding both interfaces, we can summarize by stating that distance panned and zooming attempts (both within the detail window) are mostly responsible for the differences in task-completion times between users. Moreover, we compared the zoom and pan actions within the detail window between both interface types. Analysis revealed that users panned and zoomed more often (Figure 4 a \& b) and greater distances (Figure $4 \mathrm{c} \& \mathrm{~d}$ ) using the detail-only interface. For panning and zooming distance, this difference is significant (panning distance: $M e a n=4149$ pixels and $S D=3392$ pixels compared to Mean $=2670.5$ pixels and $S D=3212$ pixels; $F(1,23)=4.837, p<0.05$; zooming distance: Mean $=2462$ level changes and $S D=316$ level changes compared to Mean $=1844$ level changes and $S D=514$ level changes; $F(1,23)=26.684, p<0.001)$. This 


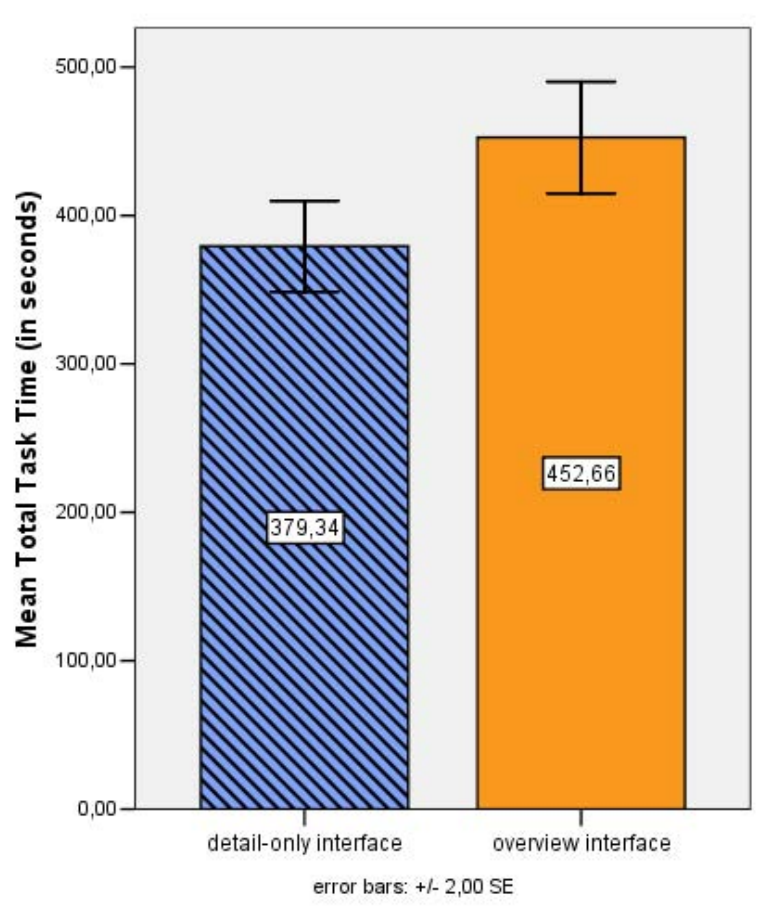

Figure 3: Comparing mean total task time between interface types

indicates that the overview window may have reduced the need for long-distance panning and zooming and, as shown by regression analysis, the panning distance is strongly correlated with task-completion time. However, since taskcompletion time for the overview interface was significantly higher, it seems as if the time needed for users to switch between views and to make up their minds about exactly how to use the overview outweighed this advantage, as predicted in our hypothesis.

\subsection{H3: Low-Spatial Subjects vs. High-Spatial Subjects}

Our third hypothesis suggested that low-spatial participants would need significantly more time to complete tasks than high-spatial participants, regardless of which interface type was used. To test this hypothesis, we first used the C-values of our spatial-ability test and tried a regression analysis with total task-completion time as dependent variable. The analysis revealed that the spatial ability only explained 1.6 percent of the variance (ANOVA result: $F(1,22)=0.358$, $\mathrm{p}=$ n.s.). For further analysis, we dichotomized our spatialability variable into low-spatial users and high-spatial users. In the process we excluded four subjects around the median, leaving ten per group. We then compared these groups with a One-Way ANOVA using spatial group as independent variable and total task time (sum for both interfaces) as dependent variable. Our high-spatial users needed on average 809.6 seconds to complete all 24 tasks while it took our lowspatial users 822.6 seconds. The difference is not significant and therefore confirms the regression analysis. Given previous research, this result was rather unexpected. We analyzed our results from the spatial-ability test and discovered that, with regard to spatial ability, our users formed a rather

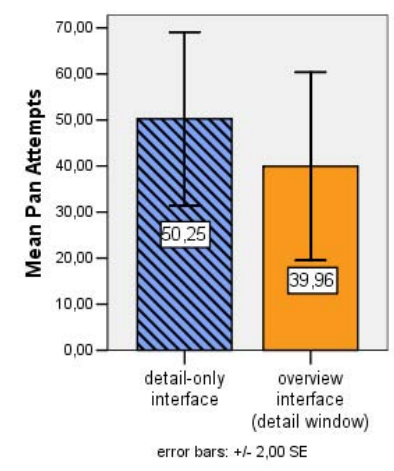

(a)

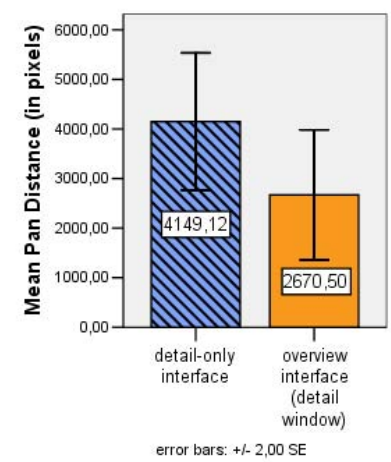

(c)

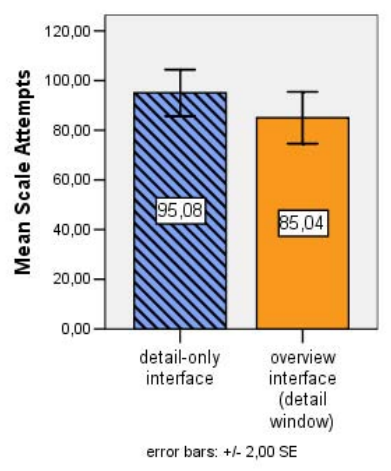

(b)

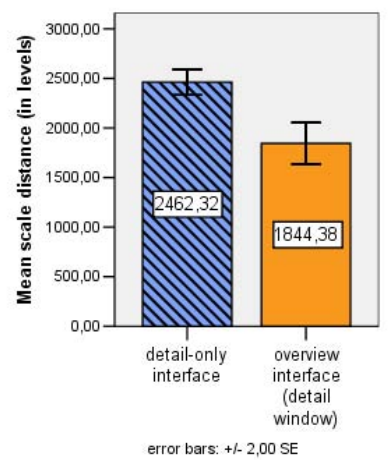

(d)
Figure 4: (a) pan attempts, (b) scale attempts, (c) pan distance, (d) scale distance - all within the detail window

homogeneous group (mean C-Value $=7.46, S D=0.977$ ). Moreover, the mean C-score of 7.46 was significantly above the population mean of $5(T(1,23)=12.326, p<0.01)$ and the mean C-Score of our low-spatial group was also significantly above the population mean (6.5 compared to 5 , $T(1,9)=6.78, p<0.01)$. Thus our subjects can be considered as "high spatial" users, which might explain why we did not find a significant difference between our high and low-spatial participants.

\subsection{H4: Overview Accommodates Spatial Dif- ferences}

Our fourth and final hypothesis follows on from the third hypothesis. We predicted that the overview interface would reduce potential differences between low and high-spatial users. Complementing the analysis in 4.3, One-Way ANOVAs revealed that there was no significant difference between low and high-spatial users for both the detail-only interface and the overview interface (detail-only: 397 seconds for low-spatial users compared to 355 seconds, $F(1,18)=$ $1.481, p=n . s$; overview: 425 seconds for low-spatial users compared to 454 seconds, $F(1,18)=0.626, p=n . s$.$) . Nev-$ ertheless, it is interesting that high-spatial users were faster than low-spatial users while working with the detail-only interface but slower while working with the overview interface. To analyze this in more detail, we conducted a RM-ANOVA with interface type as the within subjects factor and spatial group as the between subjects factor. As expected, it again revealed no significant difference between the low and high- 
spatial users (Figure 5). Between interface type and spatial group, however, we found an interesting interaction effect that corresponds to the above finding, although just beneath the 0.05 significance level $(F(1,18)=3.759, p=0.068$ n.s.). While task-completion times for low-spatial users were about equal for both interfaces (on average 397 seconds using the detail-only interface compared to 425 seconds, $F(1,9)=1.217, p=n . s$.), high-spatial users needed more time for the overview interface (on average 356 seconds using the detail only interface compared to 454 seconds). Furthermore, this difference within the high-spatial group is statistically significant $(F(1,9)=14.332, p<0.05)$. It therefore seems that the overview interface hindered high-spatial users while low-spatial users were relatively unaffected. However, there is no correlation between system preference and spatial group - four of the high-spatial users preferred the overview interface, and six the detail-only interface, meaning that the system preference of our high-spatial participants was not negatively affected by the significantly higher taskcompletion times for the overview interface.

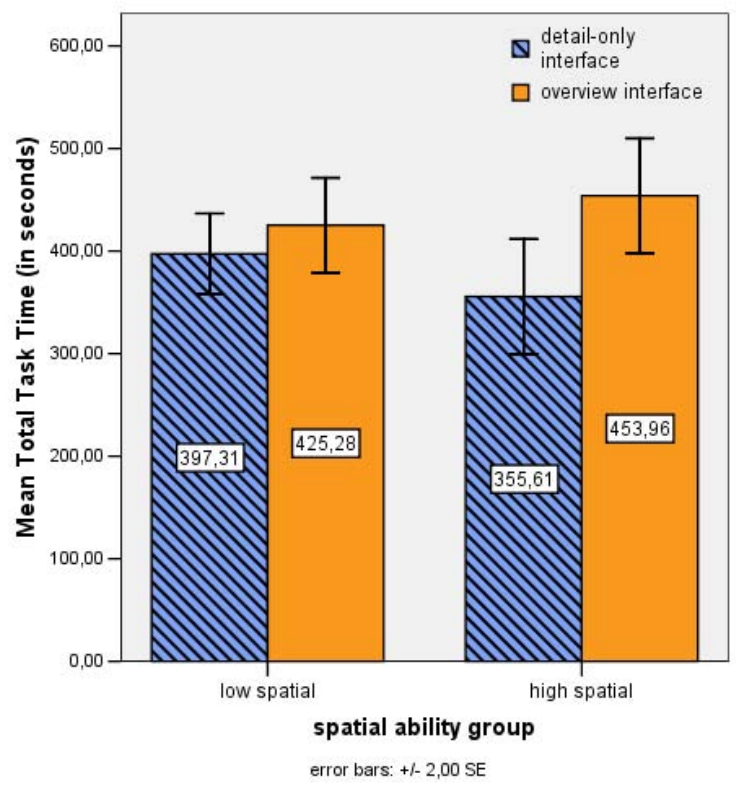

Figure 5: Comparing mean total task times between interface types separated by high and low spatial users

\subsection{Additional Analysis}

The analysis of the Attrakdiff scores revealed no significant difference between the two interfaces. Since they were very similar from a user perspective, this was a rather predictable result $(F(1,22)=2.157, p=n . s$.$) . Regarding our pre-test$ data, there was no significant difference between users who had previously used a PDA and those who had not. In fact, participants who were already familiar with PDAs were slightly slower (868 seconds for users who previously had used a PDA compared to 813 seconds, $F(1,22)=0.792, p=$ n.s.). Similar results were found for users who had previously used Google Earth. There was also no significant correlation between gender and spatial ability. The participant with the highest spatial ability was female.
After excluding a subject who never once used the pan function, the spatial ability C-Score correlated significantly with the panning distance for the detail-only interface $(r=$ $0.427, p<0.05)$ and with distance panned per attempt $(r=$ $0.487, p<0.05)$. This could indicate that low-spatial users pan in a more careful way and only small distances at a time. Regarding the error rate, we could not find a significant difference between the two interfaces. In both cases there was less than one incorrectly answered task on average. Because of the tasks selected this result was expected - more complicated tasks would have been more in the nature of memory tests for the users and would probably have covered other effects.

\section{CONCLUSIONS}

In this paper we analyzed the usability of small-screen ZUIs with and without overview with respect to individual differences in spatial ability. In an experiment that involved searching a movie database on a PDA, we found that participants were significantly faster using a detail-only ZUI compared to an overview-supported ZUI. This indicates that, on small screens, a larger detail window can outweigh the benefits gained from an overview. For high-spatial users, we assume that the performance difference is also partly due to the rich navigation cues provided by scatterplot labels. Since the overview may not offer much of an orientation benefit for those users, it thus became more of a hindrance. Low-spatial participants, on the other hand, were less affected by the overview. Their task-completion times for the overview interface turned out to be nearly equal to those for the detail-only. In this case, the overview may have actually supported the participants by preventing them from relying on their potentially incorrect mental model of the information space. However, the performance differences measured between the two user groups were not significant. We conjecture that this is mainly due to the rather small group size and cognitive homogeneity of our participants. For further research, we would recommend a broader testing approach as carried out, for example, by [24]. In that study, initially 74 individuals took the spatial-ability test. For the computer experiment, the researchers then selected 24 of the subjects who fell into the extremes of the range.

Unlike a similar study for desktop applications that had identified a strong user preference for $\mathrm{o}+\mathrm{d}$ interfaces [15], our experiment did not show a significant difference in preference between the two ZUIs. User statements suggest that not only the smaller detail view but also the difficulties experienced by the participants with zooming and panning in the overview led to a lower rating for the overview-supported interface. Interestingly, our results once again show that interface efficiency does not necessarily correlate with user satisfaction or preference.

Based on our findings, we recommend that overviews on small devices would be best used for information spaces without strong orientation cues. Moreover, designers must carefully consider the real estate trade-off as well as the increased level of visual and interaction complexity that an $\mathrm{o}+\mathrm{d}$ interface means. ZUIs, on the other hand, have been found to be an intuitive and elegant solution for visualizing data on small screens. 


\section{ACKNOWLEDGMENTS}

This work was supported by the DFG Research Training Group GK-1042 "Explorative Analysis and Visualization of large Information Spaces". We also would like to thank Dominik Morbitzer and Fabian Hallstein for their help in planning and conducting this experiment.

\section{REFERENCES}

[1] B. Allen. Information space representation in interactive systems: relationship to spatial abilities. In DL '98: Proceedings of the third ACM conference on Digital libraries, pages 1-10, New York, NY, USA, 1998. ACM Press.

[2] P. Baudisch, B. Lee, and L. Hanna. Fishnet, a fisheye web browser with search term popouts: a comparative evaluation with overview and linear view. In $A V I$ '04: Proceedings of the working conference on Advanced visual interfaces, pages 133-140, New York, NY, USA, 2004. ACM Press.

[3] B. B. Bederson, A. Clamage, M. P. Czerwinski, and G. G. Robertson. Datelens: A fisheye calendar interface for pdas. ACM Transactions on Computer-Human Interaction, 11(1):90-119, 2004.

[4] T. Büring and H. Reiterer. Zuiscat: querying and visualizing information spaces on personal digital assistants. In MobileHCI '05: Proceedings of the 7th international conference on Human computer interaction with mobile devices $\mathcal{G}$ services, pages 129-136, New York, NY, USA, 2005. ACM Press.

[5] S. K. Card, J. D. Mackinlay, and B. Shneiderman, editors. Readings in information visualization: Using vision to think. Morgan Kaufmann Publishers, San Francisco, 1999.

[6] C. Chen. Individual differences in a spatial-semantic virtual environment. Journal of the American Society of Information Science, 51(6):529-542, 2000.

[7] C. Chen, M. Czerwinski, and R. Macredie. Individual differences in virtual environments-introduction and overview. Journal of the American Society of Information Science, 51(6):499-507, 2000.

[8] C. Chen and M. P. Czerwinski. Spatial ability and visual navigation: An empirical study. The New Review for Hypertext and Multimedia, 3:40-66, 1997.

[9] M. P. Czerwinski and K. Larson. The new web browsers: They're cool but are they useful? In B. O. Thimbleby and P. Thomas, editors, People and Computers XII: Proceedings of HCI'97, Berlin, 1997. Springer Verlag.

[10] N. Dahlbäck, K. Höök, and M. Sjölinder. Spatial cognition in the mind and in the world: The case of hypermedia navigation. In Proceedings of the 18th Annual Meeting of the Cognitive Science Society University of California, San Diego, 1996.

[11] D. E. Egan. Individual differences in human-computer interaction. In M. Helander, editor, Handbook of Human-Computer Interaction. Elsevier Science Publishers B.V, 1988.

[12] D. E. Egan and L. M. Gomez. Assaying, isolating and accomodating individual differences in learning a complex skill. In D. R. F., editor, Individual Differences In Cognition, volume 2. Academic Press, New York, 1985.
[13] M. Hassenzahl, A. Platz, M. Burmester, and K. Lehner. Hedonic and ergonomic quality aspects determine a software's appeal. In $C H I$ '00: Proceedings of the SIGCHI conference on Human factors in computing systems, pages 201-208, New York, NY, USA, 2000. ACM Press.

[14] W. Horn. Leistungspruefsystem. Hogrefe Verlag fuer Psychologie, Goettingen, 1983.

[15] K. Hornbæk, B. B. Bederson, and C. Plaisant. Navigation patterns and usability of zoomable user interfaces with and without an overview. ACM Trans. Comput.-Hum. Interact., 9(4):362-389, 2002.

[16] K. Hornbæk and E. Frøkjær. Reading of electronic documents: the usability of linear, fisheye, and overview+detail interfaces. In $C H I$ '01: Proceedings of the SIGCHI conference on Human factors in computing systems, pages 293-300, New York, NY, USA, 2001. ACM Press.

[17] http://earth.google.com/.

[18] A. K. Karlson, B. B. Bederson, and J. SanGiovanni. Applens and launchtile: two designs for one-handed thumb use on small devices. In CHI '05: Proceedings of the SIGCHI conference on Human factors in computing systems, pages 201-210, New York, NY, USA, 2005. ACM Press.

[19] H. Lam and P. Baudisch. Summary thumbnails: readable overviews for small screen web browsers. In CHI '05: Proceedings of the SIGCHI conference on Human factors in computing systems, pages 681-690, New York, NY, USA, 2005. ACM Press.

[20] D. F. Lohman. Spatial ability and g. Human abilities: Their nature and assessment, pages 97-116, 1996.

[21] K. Perlin and D. Fox. Pad: an alternative approach to the computer interface. In SIGGRAPH '93: Proceedings of the 20th annual conference on Computer graphics and interactive techniques, pages 57-64. ACM Press, 1993.

[22] C. Plaisant, D. Carr, and B. Shneiderman. Image-browser taxonomy and guidelines for designers. IEEE Softw., 12(2):21-32, 1995.

[23] B. Shneiderman and C. Plaisant. Designing the user interface. Pearson/Addison-Wesley, 4. ed edition, 2005.

[24] K. M. Stanney and G. Salvendy. Information visualization: Assisting low spatial individuals with information access tasks through the use of visual mediators. Ergonomics, 38(6):1184-1198, 1995.

[25] L. L. Thurstone and T. G. Thurstone. Primary mental abilities. Psychometric Monographs, 1, 1938.

[26] K. J. Vicente, B. C. Hayes, and R. C. Williges. Assaying and isolating individual differences in searching a hierarchical file system. Hum. Factors, 29(3):349-359, 1987.

[27] K. J. Vicente and R. C. Williges. Accommodating individual differences in searching a hierarchical file system. Int. J. Man-Mach. Stud., 29(6):647-668, 1988. 